\title{
Big data analysis on online TV - Predict likes in FaceBook
}

\author{
*1 Arunkumar K, ${ }^{2}$ Dr. Devendran A \\ ${ }^{1}$ Technical Architect, ppltech, Chenai, India \\ ${ }^{2}$ Asociate Profesor, Dr. M.G.R Education and Research Instiute \\ Email: ${ }^{1}$ emailarunkumar@gmail.com, ${ }^{2}$ devendran.alagarsamy@gmail.com
}

Received: $12^{\text {th }}$ Dec 2018, Accepted $17^{\text {th }}$ March 18, Published: $30^{\text {th }}$ April 2018

\begin{abstract}
Online video is a powerful way to communicate or express any brand story, explain business value proposition clearly, and build relationships with your customers and prospects better than any other means. Recent statistics shows that including video on a landing page can increase conversion rates by $80 \%$ ? Or that $92 \%$ of mobile video consumers share videos with others. Other stats show video is shared $1200 \%$ more than both links and text combined.

Hence, video content is a powerful tool for any brand. The most popular ways to take video online are YouTube channels and Facebook pages. It is critical for business to track how their videos are performing. Both Facebook and YouTube provide enormous data on videos in their platform from video views, unique video views, the average duration of the video view and audience retention. We studied related work done in predicting no. of likes in Facebook and experimented our new predicting system on various scenarios and observed lot of interesting facts.
\end{abstract}

\section{Keywords}

Bigdata, Online Tv, Facebook, Videos

\section{Introduction}

Past decade shows an increase in the no. of video consumed over internet and hence an increase in no.of online channels. According to Cisco Forecast, video will represent $69 \%$ of all consumerbasedInternet traffic by 2017 ; this is expected to rise to $80 \%$ by 2019 . We have YouTube and Facebook having major video delivery share.

Also, we see some new online video platforms such as Vine, Instagram, and Snapchat.

To give a feel of how big these video numbers are, here are some of official numbers from YouTubeand Facebook. In January 2016, Facebookannounced that people watch around 10 million hours ofvideo a day. In February 2016, Google CEO, SundarPichai, reminded investors that YouTube'saudience watches hundreds of millions of hours of video every day.

\section{Online TV:}

Online television (or internet television) is the digital distribution of television content, such as TV shows, via the public Internet (which also caries other types of data), as opposed to dedicated terrestrial television via an over-the-air aerial system, cable television, and/or satellite television systems. The amount of data these channels put online is really huge and their success mainly depends on how these data is consumed by their subscribers. Both Facebook and you tube gives lot of data related to the views. It is crucial to analyze these data to have business insights.

\section{Related Works}

The closest work we could find in similar area is done by Kevin at al [1] in predicting the popularity of a Facebook status based on that user's personal Facebook data. Popularity, measured by the number of "likes" a post gets, is important to users because it eases the anxiety that users feel when sharing on social networking sites. Facebook is one of the core ways people express themselves, but users often struggle to decide whether their thoughts are worth sharing.

We selected features by listing all the different attributes that each status had. Beyond the status, we also listed external factors such as the time of day that the status was posted or various characteristics of the user who posted the status. Once these features were gathered for the statuses, we chose a classifier. Features used include User's number of friends, User's age, User's gender, Time the status was posted (month and time of day), Time since the last status (hours), Average number of likes, Score of the status based of the word score dictionary

\section{Works on user influence in social network:}

Work done by Hasan at al [2] on the paper 'A Survey of Link Prediction in Social Networks'. In their work they did a detailed analysis on existing methods for various link prediction systems, merits and demerits and various results were captured. Their paper is organized covering 3 important areas

1. Traditional (non-Bayesian) models, which extract a set of features to train a binary classification model.

2. Probabilistic approaches, which model the joint-probability among the entities in a network by Bayesian graphical models.

3. Linear algebraic approach which computes the similarity between the nodes in a network by rank-reduced similarity matrices.

Work done by $\mathrm{Li}$ at al [3] on the paper 'Social Network User Influence Dynamics Prediction'. Their 
paper talks majorly about the identifying the most influential users, their impact on entire social network. Their contribution is the proposal of a new model 'dynamic information propagation model based on Continuous-Time Markov Process'. This model is used to predict the influence dynamics of social network users. They compared their results with other model results.

Work done by Weng at al [4] on the paper 'Twitterrank: finding topic-sensitive influential twitterers'. Their work on identifying most influential users in 'twitter' application by a new 'Twitterrank' algorithm (which is an extension of PageRank algorithm). Their observation of $72.4 \%$ of users follow $>80 \%$ of their followers and $80.5 \%$ of users have $80 \%$ of users they are following follow them back are interesting. Also, their 'TwitterRank' algorithm outperforms related algorithms like PageRank and Topic Sensitive PageRank.

Work done by Cha at al [5] on the paper 'Measuring user influence in twitter: The million follower fallacy'. Their work focused on the experiments done on huge data from real users of twitter on 3 important measures of influences (indegree, retweets, and mentions). Their key observations are as follows

1. Popular users (high indegree) are not necessarily influential in terms of spawning retweets or mentions.

2. Most influential users can hold significant influence over a variety of topics.

3. Influence is not gained spontaneously or accidentally, but through concerted effort such as limiting tweets to a single topic.

\section{Works on Logistic regression models:}

Work done by Stephan at al [6] on the paper 'Logistic regression and artificial neural network classification models: a methodology review'. In their work they did a detailed analysis on various machine learning algorithms and compared results with 'Logistic regression' and 'Artificial neural network'. Also, they concluded with their findings on importance of both these ML models in medical data classification tasks.

Work done by Shevade at al [7] on the paper 'A simple and efficient algorithm for gene selection using sparse logistic regression'. In their work they propose a new algorithm for the sparse logistic regression problem - 'gene selection method'. Their show the result on applying this new algorithm on two real-world data sets and captured the results.

\section{Problem Statement}

Facebook provides APIs to get stats about post which has lot of data. Can we build a prediction system? for no. of views/shares/reach from history? So that users (online TV channels) has some extra tool to plan their content.

Since, the data provided by Facebook and YouTube are really huge for big channels, we must take big data processing approach to solve this problem.

\section{Experiment Setup}

We have built our prediction system using Mahout Java libraries. To keep things simple, we have taken data from a simple TV channel with 100s of video posts in Facebook for a period of 3 months, where they have fixed audience and similar marketing strategy.

We have the following data extracted from FB on every pass-through FB APIs.

a. Date and time posts - The time and date of FB posts

b. Title of post - Title of the FB post

c. Reach - No. of users who received post

d. Views - No. of users viewed post

e. Shares - No. of shares

f. Percentage of completion - Average completion of videos

g. Post content - Actual post description

\section{Final Features:}

The varying aspects for each FB posts are:
a. Actual content and category
b. Date, day, and time of post
c. Content length
d. Aspect ratio of video

We use these features to train our classifier system (supervised learning). We have built a classifier system (A system running 'Multi-Class Logistic Regression' algorithm) to consider various features. Interesting observations were made in our study as listed below.

\section{What is the differencebetween linear and logistic regression?}

In linear regression, the outcome (dependent variable) is continuous. It can have any one of an infinite number of possible values. In logistic regression, the outcome (dependent variable) has only a limited number of possible values. Logistic Regression is used when response variable is categorical in nature.In our case the categories we have chosen are no. of likes bucket (0-20 likes, 20140 likes, 401-600 likes, 601-80 likes, 801-100 likes etc.)

\section{Results And Discussion}

A. No.of views is directly proportional to no. of shares and no. of reach?

With basic intuition, we can clearly say the above statement is true. We plotted the views across shares 
and reach across shares as shown below. It confirms our intuition. Hence, we conclude that number of shares, views and reach are linearly dependent on each other. (i.e. when 1 value changes others willchange in some fixed pattern).

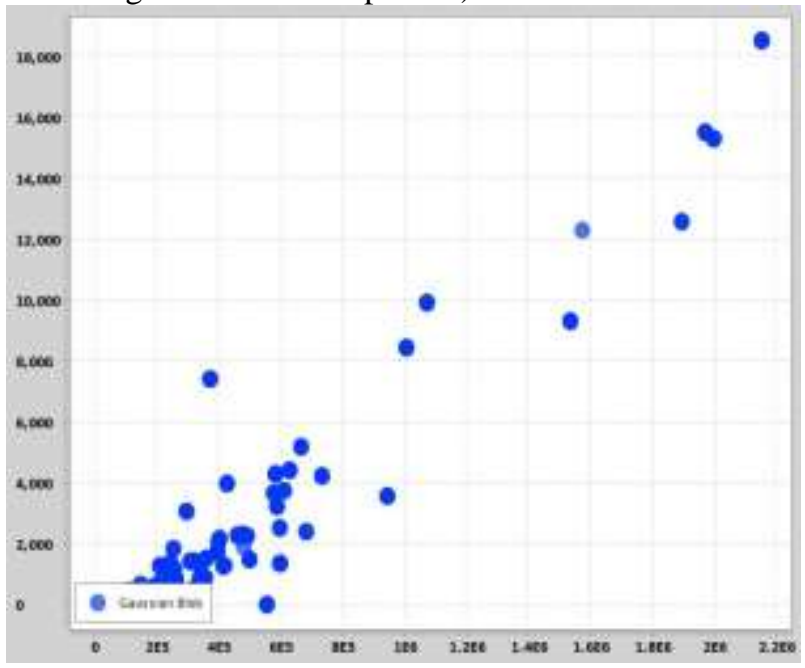

\section{B. Thursday \& Friday has most of the popular} videos

We observed most of the popular videos are being posted in Thursday or Friday (labeled as 4 and 5 in the series of 0-6 for Sunday-Saturday). This is logical as the channel's major subscribers are middle class people and watch videos for trying something new to cook.

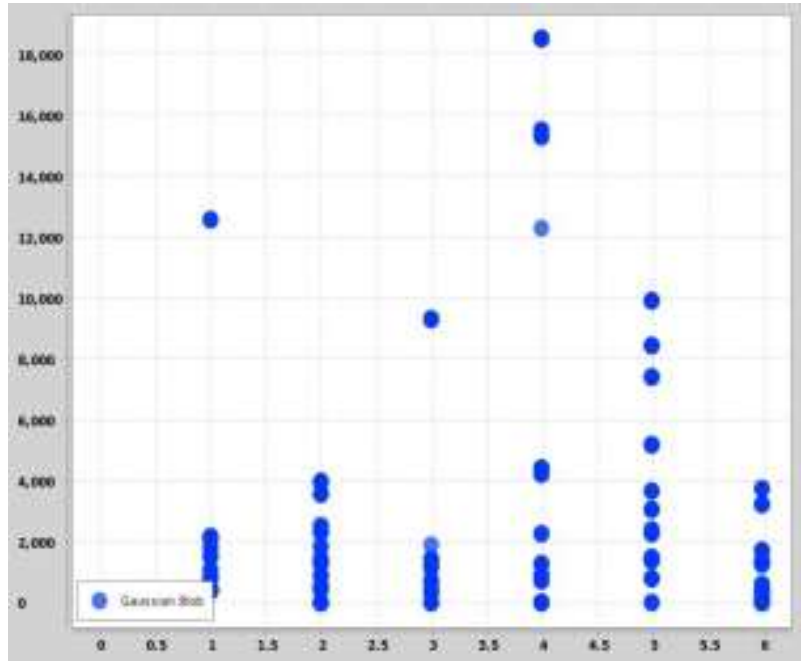

\section{4pm to 8pm has most of the popular videos}

We observed most of the popular videos are being posted around $4 \mathrm{pm}$ to $8 \mathrm{pm}$. This is logical as the channel's major subscribers are middle class people and watch videos fortrying something new to cook.

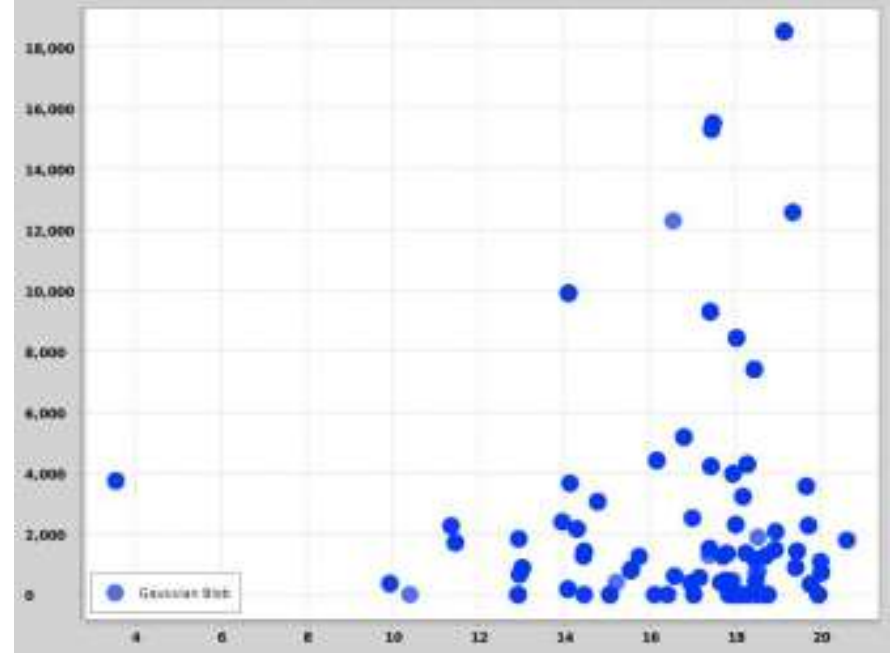

\section{All popular videos have $60 \%$ to $80 \%$ completion} view

Another key observation is that most of the popular videos are being watched have $>60 \%$ completion rate. i.e. popular videos are watched completely compared to other videos. This is an important evidence showing that content of the video plays a major role in likes and shares. Other factors like time and day also have some influence.

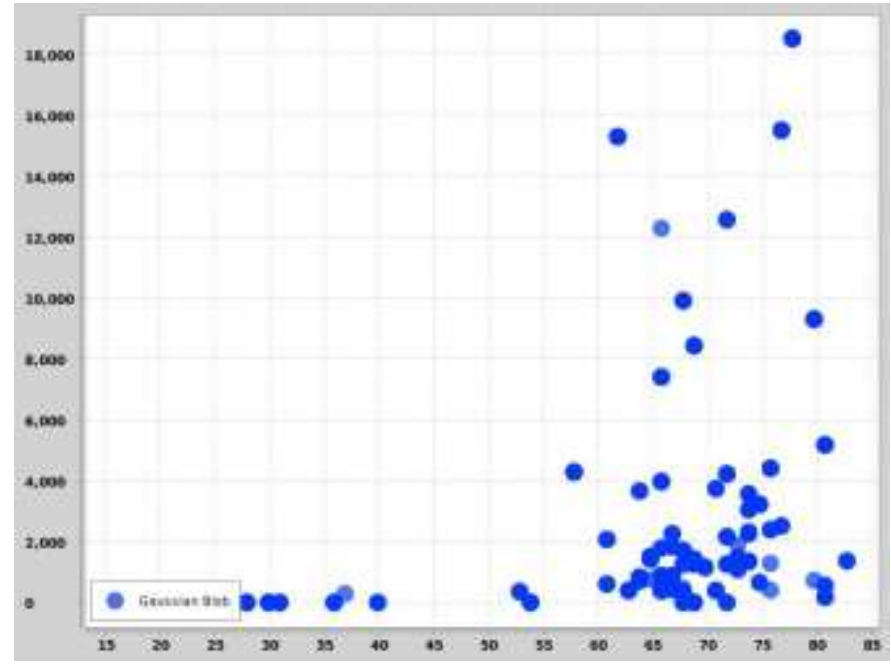




\section{Conclusion And Future Work}

Our model:

From the above observation, following is the list of varying factors and their influences.

\begin{tabular}{|l|l|}
\hline \multicolumn{1}{|c|}{ Varying Factor } & \multicolumn{1}{|c|}{ Remarks } \\
\hline Video length & $\begin{array}{l}\text { Video length is almost same for } \\
\text { all videos }(\sim 1 \text { min). Hence, no } \\
\text { influence }\end{array}$ \\
\hline $\begin{array}{l}\text { Aspect ratio of } \\
\text { video }\end{array}$ & $\begin{array}{l}\text { Aspect ratio is almost same for } \\
\text { all videos. Hence, no influence }\end{array}$ \\
\hline Day of post & $\begin{array}{l}\text { Thursday,Friday posts have } \\
\text { higher probability of success. } \\
\text { Have influence. }\end{array}$ \\
\hline Time of post & $\begin{array}{l}\text { Posts around 4pm to 8pm have } \\
\text { higher probability of success. } \\
\text { Have influence. }\end{array}$ \\
\hline Content & Have influence. \\
\hline
\end{tabular}

At this point we concluded that actual content plays a major role in popularity along with day and timeof post. Hence, we build our Regression model around these 3 parameters with more weighting on content.

We have built our recommendation system predicting the no. of likes, shares and reach for newcontents to be posted. This gave us god accuracy for selected online channel. We also observed this is not same for all the channels. Hence, we built an analysis tool and allowed the user (channel owners) to view and chose parameters for the recommendation system and view prediction accuracy.

We are planning to extend the analysis with other parameters like the category of the videos and do similar analysis for distribution in social media (like twitter and Instagram) and YouTube.

\section{References}

[1] Kevin Chen, Basil Huang, Britany Le, Northwestern University, 'Facebook Like PredictorWithin Your Friends' from 'http:/kbz.github.io/files/Final\%20report.pdf' [2] Hasan M.A., Zaki M.J. (2011) A Survey of Link Prediction in Social Networks. In: Aggarwal C. (eds) Social Network Data Analytics 2011. Springer, Boston, MA

[3] Li J., Peng W., Li T., Sun T. (2013) Social Network User Influence Dynamics Prediction. In: Web Technologies and Applications. APWeb 2013. Lecture Notes in Computer Science, vol 7808. Springer, Berlin, Heidelberg

[4] Weng, J., Lim, E.P., Jiang, J., He, Q. (2010) Twitterrank: finding topic-sensitive influential twitterers. In: WSDM 2010, pp. 261-270

[5] Cha, M., Haddadi, H., Benevenuto, F., Gummadi, K.P. (2010) Measuring user influence in twitter: The million follower fallacy. In: AAAI, ICWSM 2010

[6] Stephan Dreiseitla, Lucila Ohno-Machadob (2002) Logistic regression and artificial neural network classification models: a methodology review. In: Journal of Biomedical Informatics Volume 35, Issues 5-6, October 2002, Pages 352359

[7] S. K. Shevade, S. S. Keerthi (2003) A simple and efficient algorithm for gene selection using sparse logistic regression. In: Bioinformatics, Volume 19, Issue 17, 22 November 2003, Pages 2246-2253 